ISSN 1984-3755

Licenciado sob uma Licença Creative Commons

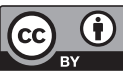

\title{
Religiosidade e saúde: experiências dos pacientes e percepções dos profissionais
}

\author{
Religiousness and health: patient's experiences \\ and professional's perceptions
}

\section{Marta Helena de Freitas}

Doutora, coordenadora do Programa de Pós-Graduação Stricto Sensu em Psicologia, Universidade Católica de Brasília, Brasília, DF - Brasil, e-mails: mhelenadefreitas@gmail.com; mhelena@ucb.br

\section{Resumo}

A partir de nossa trajetória com pesquisas e orientação de pesquisas sobre o tema, como também de um levantamento de publicações brasileiras concernentes às relações entre religiosidade e saúde nos últimos dez anos, neste trabalho realizamos uma comparação entre o que se tem constatado nas investigações com os próprios pacientes e o que se tem encontrado nos estudos e observações com os estudantes e profissionais de saúde. Voltando-se a atenção mais especificamente para os campos da psicologia, da medicina e da enfermagem, constatamos que, embora seja crescente a literatura voltada para a importância de que a assistência religiosa/espiritual seja incorporada às práticas de acolhimento e humanização em saúde, há ainda um grande hiato entre o que mostra a experiência no mundo da vida dos pacientes e a postura formal dos profissionais que os atendem nos serviços de saúde especializados. Considerando-se esse hiato e seu grande 
paradoxo, num país de população tão religiosa como o Brasil, discutimos as implicações decorrentes para a formação profissional nos diferentes campos da atenção à saúde.

Palavras-chave: Religiosidade. Espiritualidade. Saúde. Formação professional. Profissionais de saúde.

\section{Abstract}

From the own trajectory as a researcher and advisor of researches on the topic, as well from a survey of Brazilian publications concerning relationships between religiosity and health over the past ten years, in this paper we performed a comparison between what these researcher have observed in investigations with patients themselves and what they have found in studies and observations with health students and professionals. Focusing more specifically to the fields of psychology, medicine and nursing, we found that, although it is growing the literature pointing to the importance of the religious / spiritual assistance be incorporated into the practices of health hospitality and humanization, there is still a large gap between what shows the experience of the patients life and the formal posture of professionals who assist them in specialised health services. Considering this gap and its great paradox, in a religious country like Brazil, we discuss the implications for vocational training in different fields of health care.

Keywords: Religiosity. Spirituality. Health. Vocational training. Health professionals.

\section{Introdução}

“Tudo bem, graças a Deus!" ou "Vou melhorar, se Deus quiser!" são expressões que ouvimos frequentemente dos brasileiros quando questionados sobre seu estado. Tais expressões são paradigmáticas do quanto a religiosidade tem importância no mundo da vida dessas pessoas e do quanto elas mesmas a relacionam ao seu bem-estar, físico, mental ou espiritual. Mas, será que essa importância tem sido considerada e reconhecida pelos profissionais de saúde, no contexto dos diversos serviços de saúde em nosso país? Haveria algum lugar, no discurso da racionalidade 
científica que pauta a formação desses profissionais, para a escuta da fé religiosa que move seus pacientes? Ou tais afirmações tenderiam a ser consideradas como meros reflexos de perspectivas ingênuas, que nada têm a ver com o conhecimento científico que sustenta suas práticas clínicas?

Buscando algumas respostas a esses questionamentos e a outros decorrentes deles, neste trabalho procuraremos apresentar e comparar criticamente o que mostram algumas pesquisas realizadas com usuários dos serviços de saúde no Brasil e com os próprios estudantes e profissionais de saúde - psicólogos, médicos e enfermeiros - como também aquelas que se voltaram para a análise do currículo e outros elementos relativos à formação destes profissionais. Para cada um desses aspectos, iniciamos fazendo referência ao que temos encontrado em nossa própria trajetória em pesquisas e orientações de pesquisas em psicologia da religião e, na sequência, apresentaremos os resultados encontrados em várias outras pesquisas recentes sobre o tema em nosso país.

Empregamos o termo religiosidade para nos referirmos àquelas disposições humanas que levam a pessoa à capacidade de experimentar fenômenos religiosos, em seus diversos aspectos. O termo religião, por sua vez, fica reservado para se referir ao corpo social organizado em sistemas de crenças, valores e ritos religiosos ao qual uma pessoa, no cultivo de sua religiosidade, pode ou não aderir formalmente. Quanto ao termo espiritualidade, em consonância com a perspectiva fenomenológica, tomamo-lo em seu significado mais amplo, ou seja, como a capacidade de reflexão sobre si e sobre a experiência de sentido no mundo da vida e ao que lhe circunda, horizontal ou verticalmente, incluindo-se aí a dimensão religiosa. Entretanto, como a terminologia nesse campo é repleta de conexões e sobreposições, muitas vezes, nos trabalhos citados ao longo deste trabalho, os termos empregados por seus respectivos autores sofrem alguma variação, o que não impede as reflexões aqui propostas.

\section{0 que mostram as pesquisas realizadas com pacientes}

Iniciamos a desenvolver pesquisas no campo intitulado psicologia da religião há cerca de 15 anos. Ao longo desse período, tem sido 
impressionante o número de jovens pesquisadores, da graduação ao pós-doutorado, que nos procuram interessados em desenvolver ou aprofundar seus estudos acerca do papel da religiosidade na vida das pessoas. Em diálogo com experientes pesquisadores desse campo, constatamos que o mesmo vem ocorrendo com todos eles. Paralelamente, assistimos a um expressivo aumento de publicações sobre o tema, tanto em livros como em periódicos científicos, as quais eram muito raras no contexto acadêmico-científico brasileiro há menos de duas décadas. É interessante observar que, na grande maioria dessas pesquisas, ainda que se questione com frequência o papel limitador ou repressivo de algumas religiões, tem prevalecido a perspectiva de que a religiosidade tem influência positiva sobre a saúde física ou mental das pessoas, inclusive sobre aqueles que se encontram sob cuidados médicos ou psicológicos.

Em pesquisas conduzidas ou orientadas por nós, os efeitos positivos da religiosidade sobre a saúde e bem-estar na percepção das pessoas têm sido registrados, em especial, nas situações críticas ou geradoras de estresse ou preocupações específicas, tais como: doenças, violências ou perdas (DUTRA, 2008; BARBOSA, 2006; FREITAS, 2005; NASCIMENTO, 2005); velhice e perspectivas de finitude da vida (BARBOSA; FREITAS, 2009; BARBOSA, 2008; FREITAS et al., 2006); riscos ou luto por suicídio (BORGES, 2013; DENSKE, 2007) e diversas outras questões relacionadas à saúde mental (OLIVEIRA, 2008; FRANCA, 2011; NETTO, 2005). Em tais estudos, constata-se uma variedade de modos por meio dos quais o envolvimento religioso influencia positivamente a vida e a saúde das pessoas: como suporte social, como determinante de um estilo de vida mais saudável, muitas vezes funcionando como fatores de proteção; como orientadora de um sentido para a dor e para o sofrimento e consequente resiliência em seu enfrentamento; como motivadora de fé, consolo e esperança, dentre outros.

Esses resultados têm sido confirmados por várias outras pesquisas realizadas no contexto da realidade brasileira, as quais se pautam por uma diversidade de metodologias e referenciais teóricos. Selecionamos alguns dos mais recentes, priorizando aqueles que foram desenvolvidos com os próprios pacientes atendidos em hospitais gerais ou serviços de saúde mental.

Alguns dos contextos mais investigados são as unidades de oncologia ou cuidados paliativos (FORNAZARI; FERREIRA, 2010; ELIAS; 
GIGLO; PIMENTA, 2008), geriatria (DUARTE; WANDERLEY, 2011) e de atendimento a pessoas com aids (FARIA; SEIDL, 2006; MELLAGI, 2009). Em todos esses contextos, a religiosidade tem se mostrado como um elemento sempre muito presente na experiência dos pacientes, seja sob a forma de estratégias de coping, de resiliência, de superação ou ressignificação da dor, de promoção de sentido ou, mesmo, na percepção de alguns, de elemento propulsor da própria cura. As pesquisas que investigaram estratégicas de coping, como a de Faria e Seidl (2006), tendem a apontar o maior emprego do padrão positivo, em detrimento do negativo, sugerindo que a religiosidade favorece o enfoque mais voltado para o problema do que para as emoções e, ao falar dela, a maioria dos pacientes tende a apresentar emoções mais positivas do negativas.

Também o tema da oração e suas relações com os processos de coping religioso têm sido investigados em diversas situações relacionadas ao binômio saúde-doença, promoção da qualidade de vida em idosos, e outras instâncias e contextos de cuidados em saúde (ESPERANDIO; LADD, 2013). Os resultados dessas pesquisas apontam para diversos efeitos positivos, dentre os quais: redução da ansiedade, habilidade para administração do estresse, impulso para a solidariedade e a cooperação mútua, direcionamento e propósito existenciais.

Os contextos de saúde mental também têm sido focos de diversos estudos, seja o próprio hospital psiquiátrico (BALTAZAR, 2003), o Centro de Atenção Psicossocial - CAPS (SOUZA; PEREIRA, 2012) ou unidades específicas, como, por exemplo, a de idosos (DRUCKER, 2005). O que há de comum nos resultados encontrados por essas pesquisas é o fato de a saúde mental se apresentar, sempre, de uma maneira ou de outra, ligada ao fenômeno religioso. Seja reconhecendo na leitura religiosa uma tradução de melhor condição mental do idoso deprimido, seja identificando correlações entre os modos de ser religioso dos usuários e a ausência ou presença de determinados sintomas, ou ainda encontrando na religião importante fator de suporte, socialização e ressocialização dos pacientes psiquiátricos, o que não se pode negar, a partir dos resultados dessas pesquisas, é a íntima conexão entre religiosidade e saúde mental.

Por outro lado, algumas das pesquisas realizadas com os próprios pacientes mostram que, muitas vezes, a experiência religiosa dos 
mesmos não encontram eco no contexto hospitalar, seja o psiquiátrico (BALTAZAR, 2003), seja o de hospital geral (PEREIRA; ALMEIDA, 2005). Mais que isso, em ambos os casos, tais pesquisas ilustram ser comum registrar-se, no depoimento de pacientes, a constatação de uma espécie de rivalidade entre profissionais de saúde - em especial médicos psicólogos - e religiosos, na medida em que estes últimos muitas vezes acabam exercendo um papel alternativo em termos de cuidados com a saúde física ou mental, o qual tende, com frequência, a não ser reconhecido, valorizado ou mesmo aprovado pelos primeiros.

\section{0 que mostram as pesquisas realizadas com estudantes e profissionais}

Justamente em decorrência do grau de silenciamento e/ou de mobilização negativa durante muito tempo observado no âmbito técnico-científico e profissional, no que tange às questões religiosas - quanto estas, ainda que raramente, faziam-se presentes entre estudantes e profissionais da área de saúde mental — , boa parte das pesquisas que temos desenvolvido ou orientado nos últimos anos tiveram como sujeitos estudantes (FREITAS, 2007; FREITAS; ABREU E SILVA, 2003), profissionais de psicologia e/ou psiquiatria (GARCIA; FREITAS, 2013; FREITAS, 2012; ARAÚJO, 2011; SILVA, 2010; REZENDE, 2005) ou professores de psicopatologia (CARNEIRO, 2008).

Os resultados encontrados nas pesquisas com os estudantes de psicologia mostram o impacto das teorias psicológicas que, no passado e ainda hoje, tendem a "psicologizar" ou "patologizar" a religiosidade, de modo que muitos deles se mostram bastante conflitados ao longo do curso, revelando frequentes sentimentos de despreparo e insegurança para lidar com as questões religiosas de seus futuros pacientes. A grande maioria desconhece integralmente a literatura em psicologia da religião e sequer ouviram falar dos importantes escritos de grandes pioneiros dessa área, muitos deles também pioneiros da psicologia, como é o caso, por exemplo, de James, Wundt e Hall. Esse desconhecimento acaba dificultando a elaboração dos frequentes conflitos entre as crenças pessoais e os 
conteúdos assimilados ao longo de sua formação, como também ilustram os trabalhos de Ancona-Lopez (2007, 2010).

Os resultados das pesquisas realizadas com os psicólogos recém-formados mostram que eles reconhecem a presença das questões religiosas no discurso e na experiência de seus pacientes, mas se sentem despreparados para lidar com tal realidade, demonstrando receios de virem a incorrer em problemas de cunho ético. Por outro lado, as pesquisas realizadas com os psicólogos e os psiquiatras mais experientes mostram que eles, justamente por terem se deparado cotidianamente com as questões religiosas de seus pacientes e a importância delas no campo da saúde mental, acabaram desenvolvendo, a partir de estudos realizados por conta própria ou a partir de uma espécie de conciliação entre o que aprenderam da psicologia ou psiquiatria e sua própria formação religiosa, um modus operandi para lidar com tal realidade. Mas, ainda entres eles, é comum encontrar uma certa hesitação ao falar do assunto. Embora reconheçam aspectos positivos da religiosidade, enumeram também os efeitos negativos da religião como sistema, em especial no que tange a seus aspectos dogmáticos e doutrinários.

$\mathrm{Na}$ literatura recente, encontramos vários estudos realizados com equipes de saúde atuando em vários contextos, como saúde da família (SOUZA; CALDAS, 2009), oncologia (GOBATTO; ARAÚJO, 2013; ESPÍNDULA; VALLE; BELLO, 2010) ou unidades nas quais a proximidade com a morte é frequente (NASCIMENTO; ROAZZI, 2007). Tais estudos têm apontado que, mesmo quando não praticam atividades religiosas, os profissionais entrevistados reconhecem que os temas religiosos são muito presentes durante os atendimentos e têm papel relevante na vida do paciente e, muitas vezes, até mesmo em sua recuperação ou cura. Existe o reconhecimento de que muitos pacientes explicam as próprias doenças, especialmente as mentais, em função de aspectos religiosos ou espirituais; muitos deles tendem a buscar a cura nas igrejas ou na consulta a curandeiros religiosos. Em geral, os motivos dessa busca são explicados em função da concepção religiosa do paciente, mas para alguns profissionais isso se dá mais em virtude da facilidade de acesso a essas modalidades alternativas de tratamento do que pelos recursos oficiais da medicina. Por outro lado, observa-se que as crenças religiosas dos próprios profissionais influenciam o modo como lidam com a doença, a morte e a própria religiosidade dos 
pacientes e de seus familiares. Em geral, os profissionais que são religiosos tendem a aceitar melhor as explicações religiosas adotadas pelos pacientes, em especial quando compartilham as mesmas modalidades de crenças. Por exemplo, no estudo realizado por Souza e Caldas (2009), no estado da Bahia, muitos profissionais compartilham com seus pacientes a crença na influência direta dos espíritos. Por outro lado, constata-se também que, mesmo nesses casos, os profissionais desconhecem, quase completamente, modelos capazes de combinar o saber médico com a cultura, o senso comum e outras produções de conhecimento, no atendimento adequado ao paciente, em especial no que tange ao manejo dessa relação entre religiosidade e saúde física ou mental. E, em geral, todos os profissionais alegam ausência de formação para lidar de modo adequado com o tema.

Já os estudos realizados especificamente com os médicos (ALBUQUERQUE, 2006) e com profissionais de enfermagem (SALGADO; ROCHA; CONTI, 2007) mostram que, enquanto os primeiros têm maior propensão para negar a possibilidade de uma relação harmoniosa entre biocência e religiosidade, os segundos estão mais receptivos para admiti-las, mas ainda encontram grande desconforto para lidar com o tema religioso com seus pacientes.

\section{0 que mostram as pesquisas sobre a formação profissional}

Tanto nas pesquisas citadas anteriormente como em outras encontradas em recente levantamento da literatura concernente (FREITAS, [20-?]), são extremamente frequentes os depoimentos de estudantes e profissionais em saúde sobre não receberem nenhum treinamento para lidar com as expressões religiosas de seus pacientes e suas respectivas conexões com as ações em saúde e cuidados paliativos. Entretanto, ainda são praticamente inexistentes as pesquisas voltadas especificamente para análise dos currículos e dos processos de formação desses profissionais no que tange à temática. Por outro lado, encontramos duas pesquisas realizadas com os próprios professores.

Uma pesquisa exploratória realizada com professores de psicopatologia em Brasília (CARNEIRO, 2008) mostrou que os profissionais têm 
evitado tratar do assunto religião ou espiritualidade em sala de aula, embora reconheçam ser muito comum as perguntas dos alunos em torno das relações em religiosidade e saúde/doença mental. Os motivos alegados para evitar o tema é o desconhecimento de uma literatura consistente e os receios de que a discussão em sala se encaminhe para questões metafísicas ou relacionadas a crenças religiosas específicas, que não encontrem nenhum respaldo científico, fugindo aos objetivos centrais da disciplina. Essa postura é encontrada inclusive entre os que se declaram religiosos.

Outro estudo realizado com 24 professores de graduação em Enfermagem em São Paulo (BENKO; SILVA, 1996) encontrou que a maioria deles reconhece a dimensão espiritual e religiosa do ser humano e suas respectivas implicações para a saúde do paciente e para o trabalho da enfermagem. Mais de $60 \%$ dos entrevistados consideram importante o ensino da assistência espiritual no curso de graduação em Enfermagem, admitem que o tema não tem sido tratado e oferecem sugestões de como isso poderia ocorrer, seja por meio de disciplinas específicas sobre o tema, seja por meio de reflexões com os estudantes, nas disciplinas diversas, que os preparassem para lidar com a própria religiosidade, bem como com a de seus pacientes.

Por outro lado, estudos realizados a partir de uma análise de matérias veiculadas por meios que têm o aval ou são suportes para a formação acadêmica e profissional em saúde (TURRA, [20-?]; GUSSI; DYTZ, 2008; ALMEIDA; ODA; DALGALARRONDO, 2007) têm demonstrado a permanência de muitos preconceitos em relação ao tema. Indicam também a escassez, na área, de literatura específica de formação desses profissionais que explicite claramente "o que fazer", favorecendo ações concretas sobre como lidar com a religiosidade dos pacientes nos diferentes contextos de saúde física ou mental. Daí a grande relevância de se desenvolverem iniciativas de melhor instrumentalização de psicólogos, médicos e enfermeiros, dentre outras profissões, oferecendo-lhes condições para atuação condizente com as atuais políticas de assistência humanizada em saúde do país (BRASIL, 2009), as quais estabelecem como direito dos usuários em saúde: receber atendimento competente, humanizado e acolhedor, devendo os profissionais que lhes atendem não só respeitar, mas sobretudo se ater a seus valores éticos, culturais e religiosos. 


\section{Conclusões}

A presença de elementos religiosos no mundo da vida dos brasileiros é extremamente rica e de saltar aos olhos de qualquer pessoa e em qualquer ambiente, inclusive no contexto hospitalar ou outros serviços de saúde. Como vimos nas várias pesquisas relacionadas aqui, embora o profissional de saúde também esteja inserido nesse contexto (ele mesmo, muitas vezes, também cultivando sua própria religiosidade), há ainda enorme hiato entre a grande riqueza própria do munda da vida e o discurso da racionalidade científica, que tende a não se pronunciar sobre a primeira. Empregando as palavras de Turra ([20-?]), diríamos que, no contexto dos serviços de saúde, “a escassez se dá pelo silenciamento, pelo fazer não naturalizado, não autorizado institucionalmente", de modo que é comum se observar os profissionais de saúde sendo flagrados em atitudes religiosas discretas, quase às escondidas, ante seus pacientes, mas pouco ou praticamente nada disso pode ser formalizado oficialmente.

A literatura científica em psicologia da religião nesse terreno é bastante farta (KOENIG, 2013; KOENIG; KING; CARSON, 2012; PAIVA et al., 2009; NETO; LOTUFO; MARTINS, 2009; SALDAGO; FREIRE, 2008; DALGALARRONDO, 2007; MOREIRA-ALMEIDA; NETO; KOENIG, 2006; PAIVA, 1998; PARGAMENT, 1997), mas ainda pouquíssimo ou nada conhecida pelos profissionais e seus agentes formadores. Por isso, ela não chegou ainda a resultar em sistematização prática ou no alcance de um lugar institucional nos diversos serviços de saúde no país, encontrando-se raros trabalhos com propostas mais concretas nessa direção (TURRA, [20-?]; RIBEIRO, 2011; ELIAS; 2007).

Por outro lado, se as investigações sobre as formas de espiritualidade brasileiras e sobre os modos como as equipes multiprofissionais, em particular, têm-nas considerado em suas práticas cotidianas nos serviços de saúde são passos muito importantes na construção de um atendimento integral aos usuários desses serviços, elas não se mostram suficientes por si sós. Para que tais profissionais se tornem realmente capazes de incorporar formal e explicitamente, às suas práticas clínicas, preocupações não apenas procedimentais e medicamentosas, mas também questões de natureza 
cultural, ontológica e espiritual, faz-se necessária uma profunda revisão dos paradigmas que têm imperado ao longo de sua formação profissional.

$\mathrm{Na}$ revisão dos paradigmas vigentes, questões histórico-epistemológicas que marcaram o início de todas as profissões em saúde deveriam ser retomadas e estudadas/refletidas em profundidade, em vez de meramente extirpadas dos currículos, disciplinas e conteúdos ministrados ao longo da graduação. Além disso, complementarmente ao estudo da literatura científica concernente às relações entre religiosidade e saúde, estudos interdisciplinares e questões relacionadas às crenças religiosas e populares deveriam ser inseridos nos currículos, em conexão com os conteúdos dados pela racionalidade científica, de modo que tais profissionais fossem melhor preparados para lidar, de modo ético e consistente, com o que emergirá naturalmente no contato com seus futuros pacientes.

\section{Referências}

ALBUQUERQUE, L. A religiosidade no contexto hospitalar: concepções e condutas médicas diante da religiosidade do paciente. 2006. Dissertação (Mestrado) - Psicologia da Universidade de Brasília, Brasília, 2006.

ALMEIDA, A. A. S.; ODA, A. M. G. R.; DALGALARRONDO, P. O olhar dos psiquiatras brasileiros sobre os fenômenos de transe e possessão. Revista de Psiquiatria Clínica, v. 34, p. 34-41, 2007. Suplemento 1.

ANCONA-LOPEZ, M. As crenças pessoais e os psicólogos clínicos: orientação de dissertações e teses em Psicologia da Religião. In: ARCURI, I. G.; ANCONA-LOPEZ, M. (Org.). Temas em Psicologia da Religião. São Paulo: Vetor, 2007. p. 187-210.

ANCONA-LOPEZ, M. Psicólogos e enfrentamento religioso. In: SEMINÁRIO PSICOLOGIA E SENSO RELIGIOSO: ENFRENTAMENTO (COPING) RELIGIOSO E SAÚDE, 7., 2010, São Paulo. Anais... São Paulo: Instituto de Psicologia da USP; ANPEPP, 2010. p. 45.

ARAÚJO, R. C. Sentidos da afiliação e pertencimento a uma Associação de Psicólogos Cristãos: investigação fenomenológica com psicólogos clínicos. 2011. Dissertação (Mestrado em Psicologia) — Universidade Católica de Brasília, Brasília, 2011. 
BALTAZAR, D. V. S. Crenças religiosas no contexto dos projetos terapêuticos em saúde mental: impasse ou possibilidade? 2003. Dissertação (Mestrado em Saúde Pública) - Escola Nacional de Saúde Pública da Fundação Oswaldo Cruz, Rio de Janeiro, 2003.

BARBOSA, D.Aescutadofilhodesanto sobre a morte: entreosilênciodo ocidente moderno e a fala do Candomblé. 2006. Dissertação (Mestrado em Psicologia) Universidade Católica de Brasília, Brasília, 2006.

BARBOSA, K. A. Religiosidade e o enfrentamento religioso em idosos sob cuidados paliativos. 2008. Dissertação (Mestrado em Gerontologia) Universidade Católica de Brasília, Brasília, 2008.

BARBOSA; K. A.; FREITAS. M. H. Religiosidade e atitude diante da morte em idosos sob cuidados paliativos. Revista Kairós, v. 12, n. 1, p. 113-134, 2009.

BENKO, M. A.; SILVA, M. J. Pensando a espiritualidade no ensino de graduação. Revista Latino-Americana de Enfermagem, v. 4, n. 1, p. 71-85, 1996.

BORGES, M. G. P. Sentidos do suicídio na percepção de mães que perderam seus filhos. 2013. Dissertação (Mestrado em Psicologia) - Universidade Católica de Brasília, Brasília, 2013.

BRASIL. Ministério da Saúde. Portaria n. 1820, de 13 de agosto de 2009. Dispõe sobre os direitos e deveres dos usuários da saúde. Diário Oficial [da] República Federativa do Brasil, Poder Legislativo, Brasília, DF, 14 ago. 2009. Disponível em: <http://bvsms.saude.gov.br/bvs/saudelegis/gm/2009/ prt1820_13_08_2009.html>. Acesso em: $12 \mathrm{dez} .2013$.

CARNEIRO, J. D. G. Experiência espiritual e psicopatologia: a percepção de professores de psicopatologia. 2008. Monografia (Graduação em Psicologia) Universidade Católica de Brasília, Brasília, 2008.

DALGALARRONDO, P. Estudos sobre religião e saúde mental realizados no Brasil: histórico e perspectivas atuais. Revista de Psiquiatria Clínica, v. 34, 2007. Suplemento 1.

DENSKE, K. H. Experiência espiritual, psicopatologia e suicídio: dois estudos de casos no contexto da experiência religiosa cristã. 2007. Monografia (Graduação em Psicologia) — Universidade Católica de Brasília, Brasília, 2007. 
DUARTE, F. M.; WANDERLY, K. S. Religião e espiritualidade de idosos internados em uma enfermaria geriátrica. Psicologia: Teoria e Pesquisa, v. 27 n. 1, p. 49-53, 2011.

DUTRA, T. M. C. A visão da morte das pessoas com doenças sem perspectivas de cura: como o enfrentamento religioso influencia nesta questão. 2008. Monografia (Graduação em Psicologia) — Universidade Católica de Brasília, Brasília, 2008.

DRUCKER, C. Religiosidade, crenças e atitudes em idosos deprimidos em um serviço de saúde mental de São Paulo. Dissertação (Mestrado em Gerontologia) - Faculdade de Educação da Universidade Estadual de Campinas, Campinas, 2005.

ELIAS, A. C. A. et al. Programa de treinamento sobre a intervenção terapêutica "relaxamento, imagens mentais e espiritualidade (RIME) para ressignificar a dor espiritual de pacientes terminais. Revista de Psiquiatria Clínica, v. 34, p. 6072, 2007. Suplemento 1.

ELIAS, A. C. A.; GIGLIO, J. S.; PIMENTA, C. A. M. Análise da natureza da dor espiritual apresentada por pacientes terminais e o processo de sua re-significação através da intervenção relaxamento, imagens mentais e espiritualidade (RIME). Revista Latino-Americana de Enfermagem, v. 16, n. 6, 2008.

ESPERANDIO, M. R.; LADD, K. L. Oração e saúde: questões para a Teologia e para a Psicologia da Religião. Horizonte: Revista de Estudos de Teologia e Ciências da Religião, v. 11, p. 627-656, 2013.

ESPÍNDULA, J. A.; VALLE, E. R. M.; BELLO, A. A. Religião e espiritualidade: um olhar de profissionais de saúde. Revista Latino-Americana de Enfermagem, v. 18, n. 6, p. 1229-1236, 2010.

FARIA, J. B.; SEIDL, E. M. F. Religiosidade, enfrentamento e bem-estar subjetivo em pessoas vivendo com HIV/AIDS. Psicologia em Estudo, v. 11, n. 1, p. 155164, 2006.

FRANCA, F. S. L. Os sentidos do uso da Ayahuasca segundo a percepção dos próprios usuários: uma leitura fenomenológica. 2011 Dissertação (Mestrado em Psicologia) — Universidade Católica de Brasília, Brasília, 2011. 
FREITAS, M. G. M. Enfrentamento religioso por parte das idosas vítimas de violência familiar. Dissertação (Mestrado em Gerontologia) — Universidade Católica de Brasília, Brasília, 2005.

FREITAS, M. H. Religiosidade e morte na prática do profissional de saúde: implicações para a formação. In: AQUINO, T. A. A.; FREITAS, M. H.; POSSEBON, F. Morte, Religião e Psicologia. São Paulo: Fonte Editoria, [20-?]. No prelo.

FREITAS, M. H. Religiosidade e saúde mental em imigrantes: a percepção de psiquiatras e psicólogos ingleses e brasileiros. In: FREITAS, M. H.; PAIVA, G. J.; MORAES, C. C. (Org.). Psicologia da religião no mundo ocidental contemporâneo. Desafios da interdisciplinaridade. Brasília: Universa, 2012. p. 257-274. v. 2.

FREITAS, M. H. Quando o silêncio transborda, "calaboca” já morreu: religiosidade, cientificidade e formação em psicologia. In: FREITAS, M. H.; PEREIRA, O. P. (Org.). As vozes do silenciado: estudos nas fronteiras da antropologia, filosofia e psicologia. Brasília: Universa, 2007. p. 187-205.

FREITAS, M. H.; ABREU E SILVA, N. N. Crença religiosa e personalidade em estudantes de Psicologia: um estudo por meio do Questionário Pratt e do Método de Rorschach. Boletim da Academia Paulista de Psicologia, v. 23, n. 2, p. 19-24, 2003.

FREITAS, M. H. et al. Religiosidade e atitude diante da morte em idosos. 2006. Relatório final de pesquisa (Programa de Mestrado em Psicologia) Diretoria de Pesquisa da PRPGP, Universidade Católica de Brasília, Brasília, 2006.

FORNAZARI, S. A.; FERREIRA, R. E. R. Religiosidade/espiritualidade em pacientes oncológicos: qualidade de vida e saúde. Psicologia: Teoria e Pesquisa, v. 26, n. 2, p. 265-272, 2010.

GARCIA, B.; FREITAS, M. H. Profissionais de saúde mental e religiosidade: a percepção de si e de seus pacientes. In: SEMINÁRIO DE PSICOLOGIA E SENSO RELIGIOSO: MORTE, RELIGIÃO E PSICOLOGIA, 9., 2013. João Pessoa. Anais... João Pessoa: Editora Universitária UFPB, 2013. p. 59-60.

GOBATTO, C. A.; ARAUJO, T. C. C. F. Religiosidade e espiritualidade em oncologia: concepções de profissionais da saúde. Psicologia USP, v. 24, n. 1, p. 11-34, 2013. 
GUSSI, M. A.; DYTZ, J. L. G. Religião e espiritualidade no ensino e assistência de enfermagem. Revista Brasileira de Enfermagem, v. 61, n. 3, p. 377-384, 2008.

KOENIG, H. G. Spirituality in patient care: why, how, when and what? West Conchohocken: Templeton Press, 2013. (Publicada originalmente em 2002).

KOENIG, H. G.; KING, D. E.; CARSON, V. B. Handbook of religion and health. New York: Oxford University Press, 2012.

MELLAGI, A. G. 0 enfrentamento religioso em pacientes portadores de HIV/AIDS: um estudo psicossocial entre homens católicos e evangélicos. 2009. Dissertação (Mestrado) — Instituto de Psicologia da Universidade de São Paulo, São Paulo, 2009.

MOREIRA-ALMEIDA, A.; NETO, F. L.; KOENIG, H. G. Religious and mental health. Revista Brasileira de Psiquiatria, v. 28, n. 3, p. 242-250, 2006.

NASCIMENTO, M. N. F. Religião e saúde: etnografia em um grupo de RCC e um diálogo com a perspectiva junguiana. 2005. Dissertação (Mestrado em Psicologia) — Universidade Católica de Brasília, Brasília, 2005.

NASCIMENTO, A. M.; ROAZZI, A. A estrutura da representação social da morte na interface com as religiosidades em equipes multiprofissionais de saúde. Psicologia: Reflexão e Crítica, v. 20, n. 3, p. 435-443, 2007.

NETO, F. L.; LOTUFO, Z. L.; MARTINS, J. C. Influências da religião sobre a saúde mental. Santo André: ESEtec, 2009.

NETTO, R. G. Crença religiosa e sexualidade em mulheres participantes dos movimentos religiosos da Igreja Católica. 2005. Dissertação (Mestrado em Psicologia) — Universidade Católica de Brasília, Brasília, 2005.

OLIVEIRA, J. B. Conversão ao protestantismo na adolescência: três estudos de caso. 2008. Monografia (Graduação em Psicologia) — Universidade Católica de Brasília, Brasília, 2008.

PAIVA, G. J. AIDS, Psicologia e religião: o estado da questão na literatura psicológica. Psicologia: Teoria e Pesquisa, v. 14, n. 1, p. 27-34, 1998.

PAIVA, G. J. et al. Psicologia da Religião no Brasil: a produção em periódicos e livros. Psicologia: Teoria e Pesquisa, v. 25, n. 3, p. 441-446, 2009. 
PARGAMENT, K. L. The psychology of religion and coping: theory, research and practice. New York: Guilford Press, 1997.

PEREIRA, O. P.; ALMEIDA, T. M. C. Saúde e poder: um estudo sobre os discursos hegemônicos e subalternos em contextos multiculturais. Revista brasileira de crescimento e desenvolvimento humano, v. 15, n. 2, p. 91-98, 2005.

REZENDE, E. B. A esquizofrenia de uma perspectiva psicopatológica e espiritual. 2005. Monografia (Graduação em Psicologia) — Universidade Católica de Brasília, Brasília, 2005.

RIBEIRO, C. R. Proposta psicossocial para pacientes com enfermidades graves ou terminais. Revista Bioética, v. 19, n. 3, p. 819-831, 2011.

SALGADO, M. I.; FREIRE, G. Saúde e espiritualidade: uma nova visão da medicina. Belo Horizonte: Inede, 2008.

SALGADO, A. P. A.; ROCHA, R. M.; CONTI, C. C. O enfermeiro e a abordagem das questões religiosas. Revista Enfermagem UERJ, v. 15, n. 2, p. 223-228, 2007.

SILVA, A. F. S. Conexões e distinções entre experiência espiritual e psicopatologia na percepção de psicólogos recém-formados. 2010. Monografia (Graduação em Psicologia) — Universidade Católica de Brasília, Brasília, 2010.

SOUZA, I. R.; PEREIRA, J. D. M. Análise da relação entre religiosidade e saúde mental em usuários do Centro de Atenção Psicossocial I (CAPS I) de Mirinzal - MA no período de 2006-2010. In: SIMPÓSIO NACIONAL DA ASSOCIAÇÃO BRASILEIRA DE HISTÓRIA DAS RELIGIÕES, 13., 2012, São Luís. Anais... São Luís: ABHR, 2012. p. 1-15.

SOUZA, R. C.; CALDAS, N. M. Os sentidos da relação entre saúde mental e religiosidade para profissionais de saúde da família em Ilhéus - Bahia. Ciência, Cuidado e Saúde, v. 8, n. 3, p. 460-468, 2009.

TURRA, V. N. Análise das recomendações práticas para a assistência à espiritualidade no contexto da morte. In: AQUINO, T. A. A.; FREITAS, M. H.; POSSEBON, F. Morte, religião e psicologia. São Paulo: Fonte Editorial, [20-?]. No prelo. 


\section{Obras consultadas}

BERTACHINI, L.; PESSINI, L. A importância da dimensão espiritual na prática dos cuidados paliativos. Revista BioEthiKos, v. 4, n. 3, p. 315-323, 2010.

CONSELHO FEDERAL DE PSICOLOGIA - CFP. Código de ética profissional do psicólogo. Brasília: CFP, 2005.

GALINKIN, A. L. A cura no vale do amanhecer. Brasília: Technopolitik, 2008. INSTITUTO BRASILEIRO DE GeOGRAFIA E estatístiCA - IBGE. Censo Demográfico 2010: resultados gerais da amostra. Rio de Janeiro: IBGE, 2010.

PARGAMENT, K. L. God help me: toward a theoretical framework of coping for the psychology of religion. Research in the Social Scientific Study of Religion, n. 2, p. 195-224, 1990.

SÁ, A. C.; PEREIRA, L. L. Espiritualidade na enfermagem brasileira: retrospectiva histórica. O Mundo da Saúde, v. 31, n. 2, p. 225-237, 2007.

SILVA, C. R. et al. Religião e morte: qual a relação existente? Revista Enfermagem Contemporânea, v. 1, n. 1, p. 130-141, 2012.

Recebido: 09/01/2014

Received: 01/09/2014

Aprovado: 26/01/2014 Approved: 01/26/2014 